\title{
RESOLVING THE SUB-AU-SCALE GAS AND DUST DISTRIBUTION IN FU ORIONIS SOURCES
}

\author{
J. A. Eisner ${ }^{1,3}$ AND L. A. Hillendrand ${ }^{2}$ \\ ${ }^{1}$ Steward Observatory, University of Arizona, Tucson, AZ 85721, USA \\ 2 Astrophysics Department, California Institute of Technology, Pasadena, CA 91125, USA \\ Received 2011 April 25; accepted 2011 June 1; published 2011 August 9
}

\begin{abstract}
We present Keck Interferometer observations of the three prototypical FU Orionis stars, FU Ori, V1057 Cyg, and V1515 Cyg. With a spatial resolution of a few milliarcseconds and a spectral resolution of $\lambda / \Delta \lambda=2000$, our near-infrared observations spatially resolve gas and dust emission extending from stellocentric radii of $\sim 0.05 \mathrm{AU}$ to several AU. We fit these data with accretion disk models where each stellocentric radius of the disk is represented by a supergiant-type stellar emission spectrum at the disk temperature. A disk model is consistent with the data for FU Ori, although we require some local asymmetry in the disk. For V1057 Cyg the disk model does not fit our data well, especially compared to the fit quality achieved for FU Ori. We speculate that a disk wind may be contributing substantially to the observed near-IR emission in this source. The data for V1515 Cyg are noisier than the data obtained for the other two objects and do not strongly constrain the validity of an accretion disk model.
\end{abstract}

Key words: circumstellar matter - stars: individual (FU Ori, V1057 Cyg, V1515 Cyg) - stars: pre-main sequence

- techniques: interferometric - techniques: spectroscopic

\section{INTRODUCTION}

The class of FU Orionis objects was initially defined by sudden, large increases in optical-wavelength fluxes in FU Ori, V1057 Cyg, and V1515 Cyg (Herbig 1966, 1977). After these brightening events the optical spectra of these objects resemble those of low surface gravity (i.e., supergiant) stars. However for V1057 Cyg, pre-outburst spectra show that the progenitor resembled a higher surface gravity, typical $\mathrm{T}$ Tauri type star (e.g., Herbig 1958). While there are now many more members of the FU Ori class (e.g., Reipurth \& Aspin 2010; Miller et al. 2011), the three prototypes remain very well studied.

FU Ori outbursts are believed to result from periods of enhanced accretion in circumstellar disks around young stars (e.g., Hartmann \& Kenyon 1985, 1996; Zhu et al. 2007). Outbursts may be triggered by thermal instabilities (Bell \& Lin 1994), gravitational instabilities (Vorobyov \& Basu 2005), a combination of the two (Armitage et al. 2001; Zhu et al. 2009), or by interactions of a disk with a planet or nearby stellar companion (Lodato \& Clarke 2004; Clarke et al. 2005; Reipurth \& Aspin 2004; Pfalzner 2008). Spectroscopic observations of FU Ori stars have also been explained in the context of rapidly rotating stars near the edge of stability (e.g., Herbig et al. 2003). However, spectroscopic data at a range of wavelengths exhibit various features that are more consistent with disks than stellar photospheres, including highly broadened lines and poor crosscorrelation significance with single-temperature, fast-rotating giant star spectra (e.g., Greene et al. 2008; L. A. Hillenbrand et al. 2011, in preparation).

The disk model is strongly supported by interferometric observations that resolve the circumstellar emission at near-IR and mid-IR wavelengths on scales $>100 R_{\odot}$. Near- and mid-IR observations of FU Ori itself can be explained in the context of a geometrically thin accretion disk extending to stellocentric radii as small as $5.5 R_{\odot}$, inclined $55^{\circ}$ with respect to the plane of the sky (Malbet et al. 2005; Quanz et al. 2006). The high inferred disk temperature implies an accretion rate $>10^{-5} M_{\odot} \mathrm{yr}^{-1}$, substantially higher than for typical $\mathrm{T}$ Tauri stars. The disk

\footnotetext{
3 Alfred P. Sloan Research Fellow.
}

model for FU Ori supported by the interferometric data is also compatible with spectrophotometric data across a broad wavelength range (e.g., Zhu et al. 2008).

However, the disk model may not be the whole story, and other components like extended envelopes or outflows may be important in explaining the data for other FU Ori sources. The sizes of near-IR emitting regions in V1057 Cyg and V1515 Cyg are larger than expected from pure disk models, suggesting the presence of scattered light from extended structures (MillanGabet et al. 2006). Indeed, these objects also display excess long-wavelength emission that cannot be reproduced with geometrically thin disks (Kenyon \& Hartmann 1991; Green et al. 2006; Zhu et al. 2008). To explain these data, models with both disks and circumstellar envelopes have been constructed.

A key prediction of accretion disk models is differential rotation. A Keplerian disk rotates faster at smaller stellocentric radii, and shorter-wavelength spectral lines are expected to have broader line widths. Indeed, such behavior has been observed in a number of FU Ori objects (e.g., Hartmann et al. 2004; Greene et al. 2008; Zhu et al. 2009). While often difficult to reconcile with available interferometric data, alternative explanations for these line profiles have been proposed (e.g., Herbig et al. 2003). Spatially resolved near-IR spectra can determine the location of the gas directly and provide a direct test of the rotating disk model.

Recent enhancements to the Keck Interferometer (KI) now enable spatially resolved spectroscopy of the near-IR emission from circumstellar disks (Eisner et al. 2010b; Pott et al. 2010). Here we present KI observations of the three prototypical FU Ori objects: FU Ori, V1057 Cyg, and V1515 Cyg. Our data probe the entire $K$ band and are sensitive to emission from the $\mathrm{Br} \gamma$ transition of hydrogen and the rovibrational overtone transitions of $\mathrm{CO}$. We determine the relative spatial distributions of line and continuum emission in these systems, providing critical tests of circumstellar disk models.

\section{OBSERVATIONS AND DATA REDUCTION}

$\mathrm{KI}$ is a fringe-tracking long-baseline near-IR Michelson interferometer combining light from the two $10 \mathrm{~m}$ Keck apertures 
(Colavita \& Wizinowich 2003; Colavita et al. 2003). Each of the $10 \mathrm{~m}$ apertures is equipped with a natural guide star adaptive optics system that corrects phase errors caused by atmospheric turbulence across each telescope pupil, and thereby maintains spatial coherence of the light from the source across each aperture. Optical beam-trains transport the light from each Keck aperture down into a tunnel connecting the two Kecks and to a set of beam combination optics.

We used the "self-phase referencing" (SPR) mode of KI, implemented as part of the ASTrometric and phase-Referenced Astronomy (ASTRA) program (see J. Woillez et al. 2011, in preparation, for a description of the SPR mode). SPR allows deep integrations in a spectroscopic channel, which enables higher dispersion without loss of signal-to-noise. We use a grism with $\lambda / \Delta \lambda=2000$ to disperse the light. Note, however, that spectra are fully Nyquist sampled only at a resolution of $\sim 1000$. Near-IR spectral lines in FU Ori sources may be as broad as $\sim 100 \mathrm{~km} \mathrm{~s}^{-1}$ (e.g., Greene et al. 2008; Biscaya et al. 1997), and thus the spectral resolution of $\mathrm{KI}$ is reasonably matched to the observed systems.

Neon lamp spectra and Fourier transform spectroscopy are used to determine the wavelength scale for each night of observed data. While the entire $K$ band falls on the detector, vignetting in the camera leads to lower throughput toward the band edges. The effective bandpass of our observations is approximately $2.15-2.36 \mu \mathrm{m}$.

We obtained KI observations of V1057 Cyg on 2009 July 15, of V1515 Cyg on 2010 July 21, and of FU Ori on 2010 November 25 (all dates UT). On each night we also observed spatially unresolved stars of known spectral types, used to calibrate the instrumental response. The calibrator stars are HD 192985 and HD 195050 for V1057 Cyg, HD 194206 and HIP 102667 for V1515 Cyg, and HD 37147 and HD 42807 for FU Ori.

We measured fluxes, squared visibilities $\left(V^{2}\right)$, and differential phases $(\Delta \phi)$ for our targets and calibrator stars in each of the 330 spectral channels across the $K$ band provided by the grism (Figures 1-3). The data calibration is described in detail in Eisner et al. (2010b), but we provide a basic sketch here.

Normalized fluxes are calculated by dividing observed source and calibrator spectra and then multiplying by an appropriate stellar template for the calibrator (given by a Nextgen model atmosphere; Hauschildt et al. 1999). The absolute flux level and slope of the spectra are then matched to near-IR photometry from Two Micron All Sky Survey (2MASS). Error bars for the calibrated fluxes reflect channel-to-channel uncertainties, but do not include uncertainties in the overall normalization.

Squared visibilities for targets are divided by $V^{2}$ measured for calibrators. As for the fluxes, error bars reflect only channelto-channel uncertainties. We do not include the uncertainty in the overall normalization of the $V^{2}$.

Differential phases-i.e., the phase in each spectral channel relative to some fiducial channel—are calibrated by subtracting the $\Delta \phi$ measured for the calibrator stars. The broadband slope and curvature of the measured differential phases are altered by instrumental effects. The slope is altered by errors in group delay tracking, and curvature in the phase versus wavelength may be introduced by atmospheric water vapor turbulence and dispersion and refraction during internal beam transport (see Pott et al. 2010). Hence we cannot attribute any broadband slope or curvature in the data to the observed source. We therefore remove the slope and curvature seen across our bandpass from the differential phase data, as described in Pott et al. (2010).

\section{GENERAL FEATURES OF THE DATA}

All objects in our sample show strong absorption in the spectral regions corresponding to the $\mathrm{CO}$ rovibrational bandheads, $v=2 \rightarrow 0,3 \rightarrow 1$, and $4 \rightarrow 2$ (Figures $1-3$ ). The rest wavelengths of these three bandheads are 2.2936, 2.3227, and $2.3527 \mu \mathrm{m}$, respectively.

FU Ori and V1057 Cyg also show dips in $V^{2}$ at the wavelengths corresponding to these spectral features. V1515 Cyg may show similar dips relative to the $V^{2}$ seen in adjacent channels corresponding to continuum emission, but these dips are not significant given the noise in these data.

Lower $V^{2}$ generally means more resolved emission. However, because the $\mathrm{CO}$ appears in absorption, the dips in $V^{2}$ actually indicate that the $\mathrm{CO}$ distribution is more compact than the continuum emission. This can be understood by considering a compact, hot region of the disk that includes $\mathrm{CO}$ absorption, and a cooler, continuum-emitting region at larger radii. At wavelengths where $\mathrm{CO}$ absorbs emission from the hot, compact component, the flux-weighted size of the two regions moves to larger stellocentric radii. Thus, the compact $\mathrm{CO}$ absorption leads to a larger measured size at these wavelengths and hence a dip in $V^{2}$.

The observed differential phases indicate a centroid offset between the continuum emission and the CO-absorbing material. This offset is most obvious for FU Ori and marginally significant for V1057 Cyg. For V1515 Cyg the data are too noisy to argue the presence or absence of such an offset. Differential phase offsets in the $\mathrm{CO}$ bandheads indicate that the annuli of the disk that have appropriate temperatures for $\mathrm{CO}$ absorption in their spectra must have centroids that differ from annuli that are too hot for $\mathrm{CO}$ to exist or cool enough that they produce continuum emission.

The spectra for all objects show an absorption feature at the central wavelength of $\mathrm{Br} \gamma, 2.1662 \mu \mathrm{m}$. In addition, the spectra show hints of some emission on the red side of the line. These spectral shapes are consistent with P Cygni profiles seen in other hydrogen lines (e.g., Herbig et al. 2003).

One object-V1057 Cyg - also shows a significant dip in $V^{2}$ in the $\mathrm{Br} \gamma$ spectral region. Since the line is predominantly seen in absorption, the same argument applied to $\mathrm{CO}$ above suggests that the $\mathrm{Br} \gamma$ absorption occurs in more compact regions than the continuum emission. The $\mathrm{Br} \gamma$ feature is more prominent in the spectrum of V1057 Cyg than in FU Ori or V1515 Cyg; the higher line-to-continuum ratio of $\mathrm{Br} \gamma$ in this object may explain why the $V^{2}$ dip is only seen here.

The CO bandhead features are much broader in V1057 Cyg than in other objects in our sample. These broad features do not appear to be due to instrumental effects. During the night of our V1057 Cyg observations, we observed known CO-emitting objects immediately before and after V1057 Cyg. Neither of these objects shows such broad features (see Eisner et al. 2010a). We also verified that the appearance of the CO features in V1057 Cyg persists across multiple observations and for different calibrator stars. Thus the broadening seen in the $\mathrm{CO}$ lines of V1057 Cyg appears to be intrinsic to the source.

\section{MODELING}

In this section, we attempt to create a physically motivated circumstellar disk model to explain the measured fluxes, squared visibilities, and differential phases. In our modeling, we assume that the emission from our targets arises entirely from 

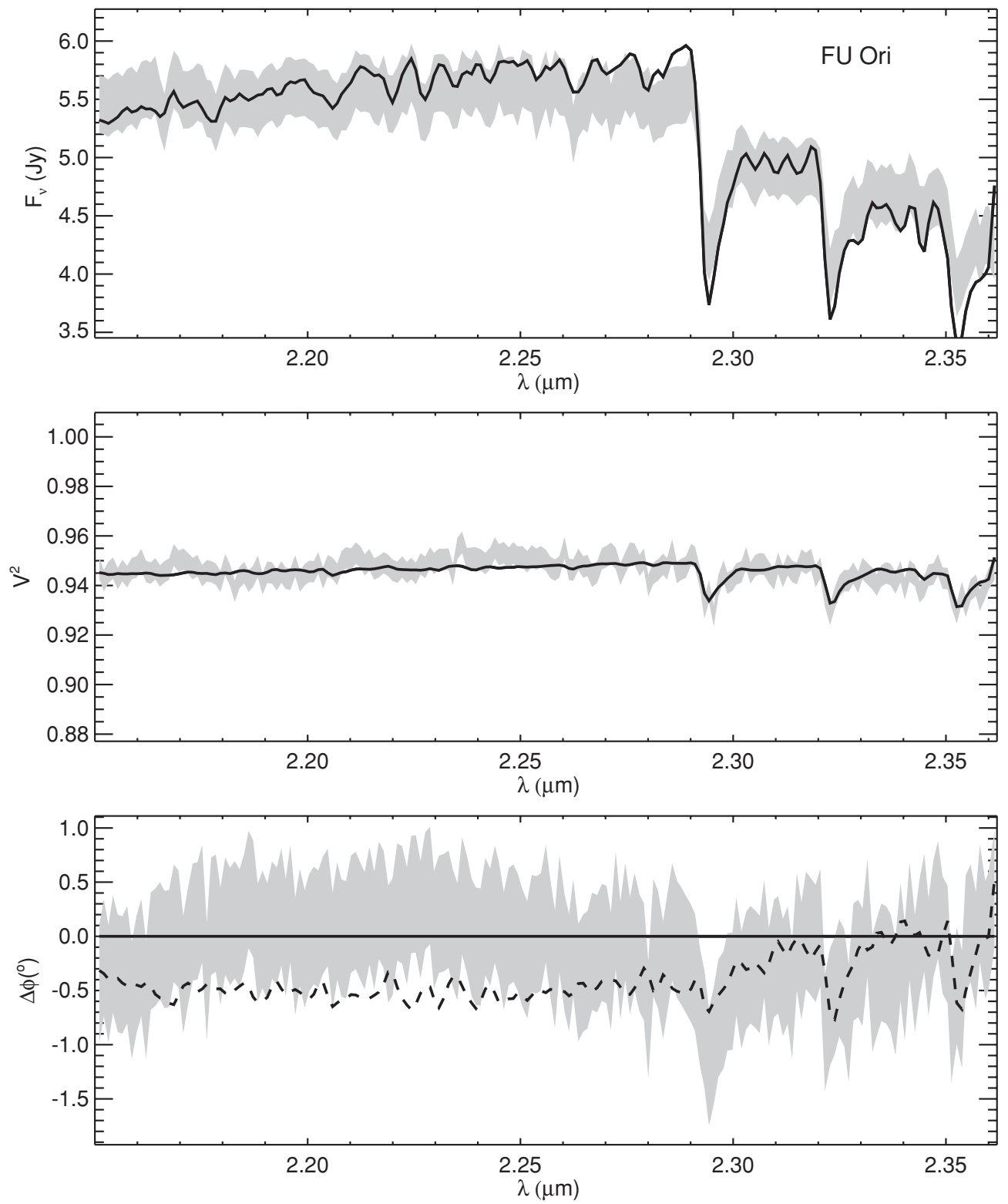

Figure 1. Fluxes, squared visibilities, and differential phases observed for FU Ori (data and $1 \sigma$ uncertainties shown with gray shaded regions). We also plot the fluxes, $V^{2}$, and $\Delta \phi$ predicted by our best-fit disk model (solid curves). The best-fit model predicts a flat differential phase across the observed bandpass and misses the significant deviations in $\Delta \phi$ seen in and out of the CO bandheads. A model exhibiting a centroid offset with $\Delta \theta_{\max }=0.8$ mas (dashed curve) fits the differential phases in the $\mathrm{CO}$ bandhead region well.

circumstellar material. In previous modeling of FU Ori sources spectrophotometric data have been fitted well with no contribution from a compact stellar photosphere (e.g., Zhu et al. 2008). We therefore do not require any correction for unresolved flux from the central star.

We begin with a geometrically thin disk model. We assume that the disk spans a range of radii from $R_{\text {in }}$ to $R_{\text {out }}$ and has a temperature profile $T=T_{\text {in }}\left(R / R_{\text {in }}\right)^{\alpha}$. We divide the disk up into a series of annuli and assume that the emergent spectrum of each annulus is that of a supergiant star. For FU Ori stars, this is a reasonable assumption since they are heated primarily from accretion energy released near the midplane. Disk atmosphere models have been constructed specifically for FU Ori systems, and these do provide better fits to detailed spectrophotometric data than models based on stellar atmospheres (e.g., Hartmann et al. 2004). We are, however, not concerned with this level of detail here and deem our models based on giant star atmospheres to be sufficient.

For annular temperatures between 1700 and 10,000 K, we use emergent spectra for stars with $\log g=0$ (Hauschildt et al. 1999). (In practice, the maximum temperature is typically between 4000 and $6000 \mathrm{~K}$.) For $T<1700 \mathrm{~K}$, we assume that the emission is a simple blackbody. The emergent spectrum is rotationally broadened by the Keplerian velocity expected for each annulus, $v \sin i=\sqrt{G M / R} \sin i$. We take $M=0.5 M_{\odot}$ for our objects; this is compatible with the dynamical mass of $0.3 \pm 0.1 M_{\odot}$ for FU Ori determined by Zhu et al. (2009) and the pre-outburst spectral type of V1057 Cyg. The spectrum of the disk model is given by the sum of fluxes over all annuli.

The visibility of each annulus is given by the visibility of a uniform ring (e.g., Eisner et al. 2004). The visibilities for the entire disk are computed as the flux-weighted average of the 

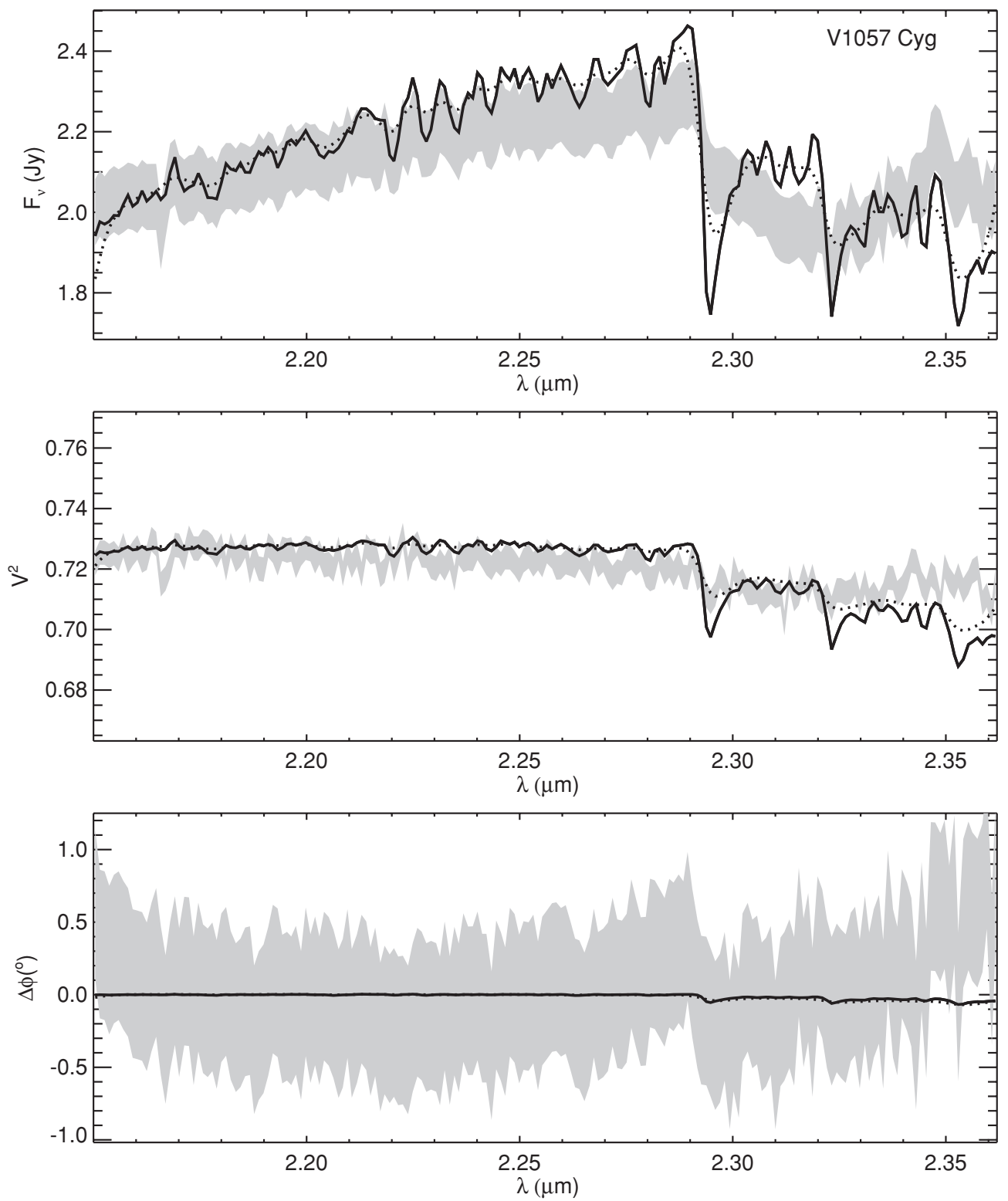

Figure 2. Fluxes, squared visibilities, and differential phases observed for V1057 Cyg (data and $1 \sigma$ uncertainties shown with gray shaded regions). We plot the fluxes, $V^{2}$, and $\Delta \phi$ predicted by our best-fit disk model with solid curves. We also show the predictions of a model where we scaled the velocities up so that the CO lines are rotationally broadened by $\sim 500 \mathrm{~km} \mathrm{~s}^{-1}$ (dotted curves) to illustrate how a disk wind might appear different than a rotating disk. The model with higher velocities produces a reduced $\chi^{2}$ of 1.3 , compared to 1.9 for the baseline disk model.

annular visibilities:

$$
V_{\text {disk }}^{2}=\left(\frac{\sum F_{\text {annulus }} V_{\text {annulus }}}{\sum F_{\text {annulus }}}\right)^{2} .
$$

The differential phase is related to the centroid offset. For each annulus, the differential phase is

$$
\Delta \phi_{\text {annulus }} \approx 2 \pi r_{u v} \Delta \theta_{\text {annulus }},
$$

where $r_{u v}$ is the $u v$ radius (see, e.g., Eisner et al. 2004) and $\Delta \theta_{\text {annulus }}$ is the centroid offset. This approximation is valid as long as the emission is not well-resolved at the angular resolution of the observations. The differential phase signal for the entire model is (see, e.g., Eisner et al. 2010b)

$$
\Delta \phi_{\text {disk }}=\frac{\sum \Delta \phi_{\text {annulus }} V_{\text {annulus }} F_{\text {annulus }}}{\sum V_{\text {annulus }} F_{\text {annulus }}} .
$$

As described above, the slope and curvature of the observed, broadband differential phase are altered by instrumental effects. We therefore remove broadband slope and curvature from both observed and modeled $\Delta \phi$.

The simple, geometrically thin disk model considered above would not produce centroid offsets. However, if we allowed the disk to be somewhat asymmetric, certain disk radii could have centroids offset from those of other disk radii. We allow for the possibility of such asymmetry by including a centroid offset that changes linearly with disk radius. This aspect of the model is described by a single free parameter that gives the amplitude of this offset. We denote this amplitude by $\Delta \theta_{\max }$.

To account for the possibility that some of the near-IR emission arises from more spatially extended regions-for example, in remnant envelopes (as implied by the results of Millan-Gabet et al. 2006) —we include a scattered light contribution in our models. While there may also be thermal emission from a 

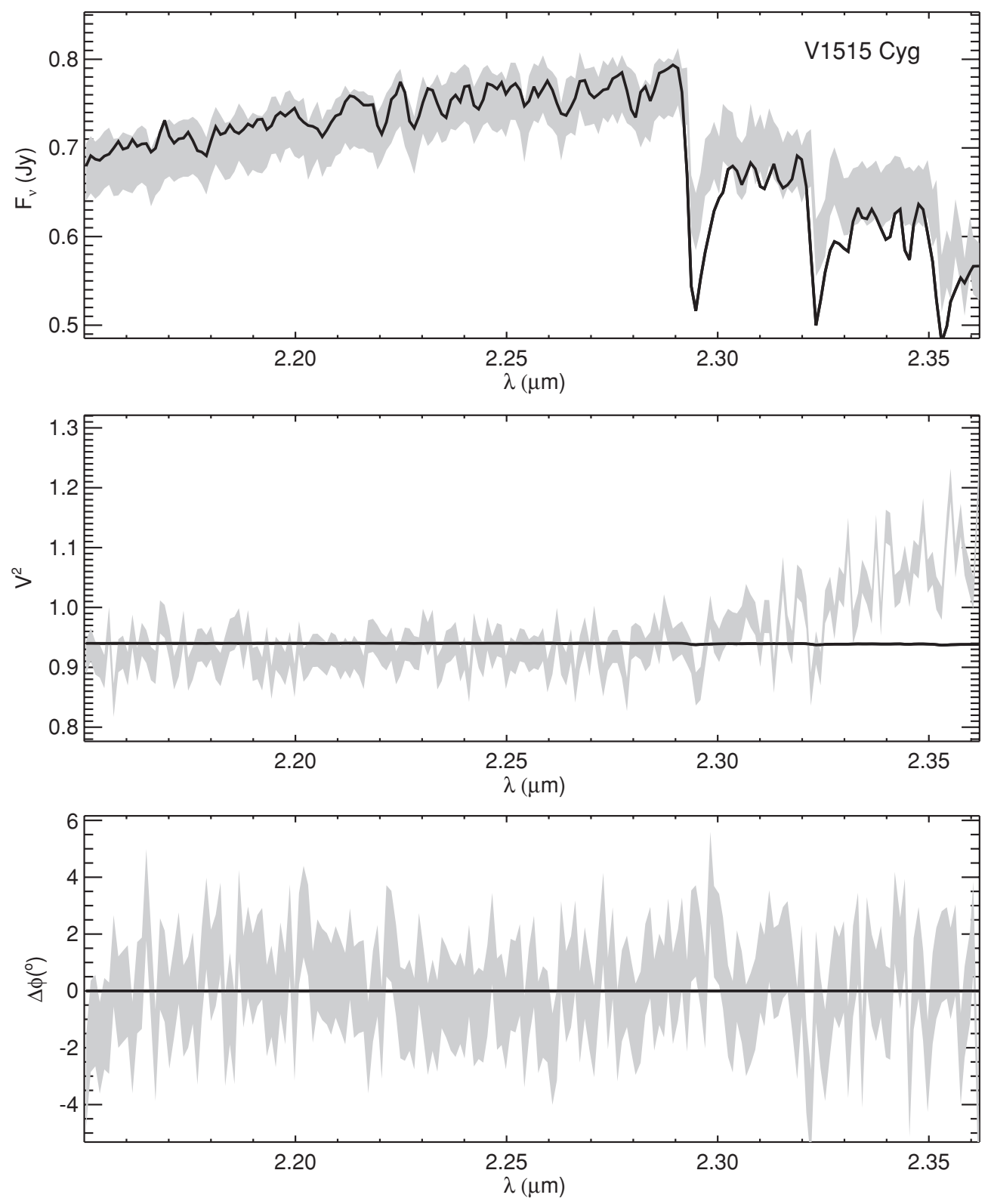

Figure 3. Fluxes, squared visibilities, and differential phases observed for V1515 Cyg (data and $1 \sigma$ uncertainties shown with gray shaded regions). We also plot the fluxes, $V^{2}$, and $\Delta \phi$ predicted by our best-fit disk model (solid curves). Note that the $V^{2}$ values greater than unity, seen for $\lambda \gtrsim 2.33 \mu \mathrm{m}$, are unphysical and are probably caused by lower signal-to-noise near the band edges.

more extended component, the cooler temperatures at large stellocentric radii would produce a very small fractional flux in the near-IR. We assume that the extended, scattered emission has the same spectrum as the disk and is sufficiently extended so that it is completely resolved $\left(V^{2}=0\right)$ in our observations. Writing the fractional scattered flux, $f=F_{\text {scat }} / F_{\text {disk }}$, the model fluxes and visibilities are modified to

$$
\begin{gathered}
F_{\text {model }}=F_{\text {disk }}(1+f), \\
V_{\text {model }}^{2}=V_{\text {disk }}^{2}(1+f)^{-2} .
\end{gathered}
$$

Finally, we include two scaling factors in the model that take into account additional uncertainties not reflected in the error bars for the data. The first is a constant scaling in the overall flux level, $X_{\text {flux }}$. This scaling accounts for uncertainties in the flux calibration beyond the assumed channel-to-channel errors, including uncertainties in the 2MASS photometry used to normalize the spectra, or source dimming between the photometric and interferometric observations. We also include a constant scaling in the overall $V^{2}$ normalization, $X_{V^{2}}$, to account for overall calibration uncertainties beyond the channelto-channel uncertainties reflected in the plotted error bars. These two scale factors may also absorb uncertainty in the assumed source distances (Table 1). It turns out that these scaling factors are near unity in our best-fit models and hence are not crucial to the fitting process; we include them for completeness.

For FU Ori, the disk inclination and position angle have been measured using previous interferometric observations. In the near-IR, the inclination and position angle were estimated as $\sim 55^{\circ}$ and $47^{\circ}$, respectively (Malbet et al. 2005). In the mid-IR, the inclination and position angle were found to be $\sim 55^{\circ}$ and $100^{\circ}$, respectively (Quanz et al. 2006). We adopt the near-IRdetermined values here. For V1057 Cyg and V1515 Cyg, no 
Table 1

Targets and Observations

\begin{tabular}{|c|c|c|c|c|c|c|c|}
\hline Source & $\begin{array}{c}\alpha \\
(\mathrm{J} 2000)\end{array}$ & $\begin{array}{c}\delta \\
(\mathrm{J} 2000)\end{array}$ & $\begin{array}{c}d \\
(\mathrm{pc})\end{array}$ & $\begin{array}{c}\text { Observation Date } \\
\text { (UT) }\end{array}$ & $\begin{array}{c}B_{\text {proj }} \\
(\mathrm{m})\end{array}$ & $\begin{array}{c}B_{\mathrm{PA}} \\
\left({ }^{\circ}\right)\end{array}$ & Calibrators \\
\hline FU Ori & 054522.36 & +090412.30 & 412 & 2010 Nov 25 & $82-84$ & $53-55$ & HD 37147, HD 42807 \\
\hline V1057 Cyg & 205853.73 & +441528.54 & 600 & 2009 July 15 & $78-81$ & $46-53$ & HD 192985, HD 195050 \\
\hline V1515 Cyg & 202348.02 & +421225.80 & 1000 & 2010 July 21 & $78-80$ & $45-48$ & HD 194206, HIP 102667 \\
\hline
\end{tabular}

Notes. $B_{\text {proj }}$ and $B_{\mathrm{PA}}$ are the projected length and position angle, respectively, of the KI baseline on the sky during our observations. The distance to FU Ori is based on trigonometric parallax measured to the Orion region (Reid et al. 2009). V1057 Cyg lies in the North American Nebula, for which the distance has been estimated to be 600 pc (Laugalys \& Straižys 2002). V1515 Cyg resides in the Cyg R1 region, for which the distance has been estimated as 1000 pc (Racine 1968).

such geometrical measurements are available, and so we leave inclination and position angle as free parameters in our models. We cannot, however, constrain these well with the limited $u v$ coverage of our observations.

Given the large numbers of free parameters, we fit our models to the data using a nonlinear gradient-fitting algorithm based on the Levenberg-Marquardt method. Such algorithms may fall into local minima in the $\chi^{2}$ surface, and so initial parameter guesses and step sizes for gradient searches influence the results. We have selected step sizes that ensure that all free parameters are varied during the fitting process. However, we are hesitant to claim that the "best-fit" models we determine are the true, global best fits.

To improve the signal-to-noise ratio in the fitting, we only use data with wavelength between 2.15 and $2.36 \mu \mathrm{m}$ to constrain the models. Data at the band edges are substantially noisier because of instrumental vignetting and contributions from ambient water vapor (Pott et al. 2010; Woillez et al. 2011).

\section{MODELING RESULTS}

\subsection{FU Ori}

Our best-fit disk model for FU Ori is shown in Figure 1. The model fits quite well in this wavelength region (reduced $\chi^{2}=0.5$ ). While the modeled differential phases do not match the observations perfectly, this is likely due to imperfectly subtracted slope and curvature (which are instrumental effects). Indeed, residual curvature of the differential phase data forces the best-fit model toward a flat differential phase versus wavelength.

If we consider the differential phase data only over the narrower spectral region containing the $\mathrm{CO}$ bandheads (where broadband slope and curvature effects are less important), we can easily construct a model that agrees with the data well. In particular, we use the best-fit model parameters for FU Ori listed in Table 2, increase the value of $\theta_{\max }$, and subtract a constant phase offset. Note that $\theta_{\max }$ has no impact on the fluxes or $V^{2}$ values and so we are free to modify its value in order to better fit the differential phases. The dashed line in Figure 1 shows that a model with a large centroid offset, $\theta_{\max }=0.8$ mas, produces a differential phase signature that agrees well with the observations in the $\mathrm{CO}$ bandhead region.

The differential phase signature seen for FU Ori thus cannot be explained with a simple, geometrically thin disk model. Rather we require a centroid offset that depends on stellocentric radius. A disk warp can obscure part of the outer regions of a disk while leaving the inner regions unobscured (or vice versa). The centroid offsets implied by our data suggest that the FU Ori inner disk may be significantly warped. In our model, this warp occurs over stellocentric radii spanning 0.07-0.49 AU.
Table 2

Disk Model Fits

\begin{tabular}{lccc}
\hline \hline & FU Ori & V1057 Cyg & V1515 Cyg \\
\hline$\chi_{\mathrm{r}}^{2}$ & 0.5 & 1.9 & 2.6 \\
$R_{\text {in }}(\mathrm{AU})$ & $0.07 \pm 0.02$ & $0.03 \pm 0.07$ & $0.03 \pm 0.11$ \\
$R_{\text {out }}(\mathrm{AU})$ & $0.49 \pm 0.03$ & $3.54 \pm 3.14$ & $1.02 \pm 0.20$ \\
$\alpha$ & $-0.95 \pm 0.10$ & $-0.50 \pm 0.03$ & $-0.72 \pm 0.13$ \\
$T_{\text {in }}(\mathrm{K})$ & $5529 \pm 779$ & $4054 \pm 1169$ & $5973 \pm 1548$ \\
$f$ & $0.02 \pm 0.01$ & $0.11 \pm 0.01$ & $0.03 \pm 0.03$ \\
$\Delta \theta$ (mas) & $0 \pm 0$ & $36 \pm 104$ & $0 \pm 0$ \\
$X_{V^{2}}$ & $1.1 \pm 0.1$ & $1.0 \pm 0.1$ & $1.0 \pm 1.0$ \\
$X_{\text {flux }}$ & $1.0 \pm 0.1$ & $1.0 \pm 0.1$ & $1.0 \pm 1.0$ \\
\hline
\end{tabular}

Notes. Parameters are defined in Section 4. While we also fitted inclination and position angle for V1057 Cyg and V1515 Cyg, these very poorly constrained by our data and so we have not listed the fitted values here.

Disk winds may also cause centroid offsets that vary as a function of stellocentric radius (e.g., Calvet et al. 1993; Hartmann \& Calvet 1995). Since all of the sources in our sample are known to be losing mass via winds (e.g., Croswell et al. 1987), this may be an alternative explanation for the differential phase signatures seen in the data. In any event, the large differential phases observed across the spectral region including $\mathrm{CO}$ bandhead absorption indicate substantial geometric asymmetry in the underlying emission from FU Ori.

The outer radius derived for FU Ori is $\sim 0.5 \mathrm{AU}$, similar to the $\sim 0.5-1$ AU value derived in the spectral energy distribution (SED) modeling of Zhu et al. (2007). This likely represents the outer radius of the hot, high accretion rate, inner disk component. As argued in Zhu et al. (2007), this implies that the high accretion rate in FU Ori is probably not caused by pure thermal instability, but rather gravitational or magneto-rotational instability. Note that a larger $R_{\text {out }}$ value is not required to fit the broadband SED, and our model fits photometry out to $\lambda \approx 10 \mu \mathrm{m}$ well. To fit longer-wavelength photometry requires an additional model component such as a flared disk (e.g., Zhu et al. 2008).

\subsection{V1057 Cyg}

For V1057 Cyg the disk model does not fit the CO bandhead features well (Figure 2). The reduced $\chi^{2}$ residuals of the fit to our $K$-band data are 1.9. The discrepancy is due to the extremely broad $\mathrm{CO}$ overtone absorption features. For an assumed $0.5 M_{\odot}$ central object, the Keplerian velocities do not exceed $\sim 100 \mathrm{~km} \mathrm{~s}^{-1}$, and hence the modeled CO bandheads are not broadened significantly.

A higher inclination would yield higher $v \sin i$ values, but would also lower the modeled flux and make it difficult to fit the observations. Moreover, even for $i=90^{\circ}$ the synthetic spectra 
are still not as broad as the data. A higher central mass can also increase the Keplerian velocities. However, $M_{*}>50 M_{\odot}$ is required to produce the $\sim 500 \mathrm{~km} \mathrm{~s}^{-1}$ broadening seen in the data. A stellar mass this high is not (remotely) compatible with the pre-outburst, K-spectral type determined for V1057 Cyg (Herbig 1958).

Such high velocities could, however, be created by a rotating disk wind (e.g., Calvet et al. 1993). V1057 Cyg is known to possess a variable, high-velocity wind (Herbig 2009; Croswell et al. 1987). If this wind dominated the near-IR absorption during the epoch of our observations, it may explain the broad $\mathrm{CO}$ absorption features in our data. While previous observations of V1057 Cyg found the CO bandheads to be narrower than inferred here (Carr et al. 1987; Hartmann \& Kenyon 1987), the $\mathrm{CO}$ overtone absorption spectra show substantial variability (Biscaya et al. 1997). When the wind is more dominant, the accretion luminosity generated in the disk midplane may be absorbed by $\mathrm{CO}$ (and other matter) with radial velocities substantially higher than Keplerian.

Rotating disk-wind models are substantially more complex than the simple disk models considered here. Rather than creating an accurate wind model, we demonstrate the overall features of such a model with a simple modification of our baseline disk model. Specifically, we scale up the Keplerian rotation profile to account for the possibility of higher radial velocities due to outflowing motions.

Figure 2 shows that a model with substantially higher radial velocities does indeed fit the data better than the simple disk model. Here we scaled the disk velocity structure up (arbitrarily) by a factor of 30, so that the $\mathrm{CO}$ lines are broadened by $v \sin i \sim 500 \mathrm{~km} \mathrm{~s}^{-1}$. This model fits the data substantially better than the Keplerian disk model considered above; the highvelocity model produces a reduced $\chi^{2}$ value of 1.3 . While the fit deviates from the data for the $\mathrm{CO} v=4 \rightarrow 2$ transition, this deviation may be due, in part, to lower signal-to-noise of the data toward the band edge.

Our best-fit disk model also fails to reproduce the $V^{2}$ dip in the $\mathrm{Br} \gamma$ spectral region observed for V1057 Cyg. Because the inner disk temperature is $\approx 4000 \mathrm{~K}$ for our best-fit disk model, there is essentially no $\mathrm{Br} \gamma$ absorption in the model spectra. To explain the $\mathrm{Br} \gamma$ feature seen in the data would require an additional model component with a higher temperature. This component would also have to be reside at very small stellocentric radii $(\lesssim 0.1 \mathrm{AU}$, which is more compact than the distribution of COabsorbing material) to produce the observed dip in $V^{2}$. Across the $K$ band, a simple disk model is not an obvious match to our observations of V1057 Cyg.

\subsection{V1515 Cyg}

The $V^{2}$ and $\Delta \phi$ observed for V1515 Cyg are noisy compared to the data for the other two objects in our sample. V1515 Cyg is more than a magnitude fainter than V1057 Cyg, and $\sim 3$ mag fainter than FU Ori, and so more noise is expected. V1515 Cyg is near (but within) the brightness limit of the SPR mode, beyond which signal-to-noise degrades significantly (Woillez et al. 2011). However, lower throughput near the band edges means that the signal-to-noise is degraded more in the spectral region including $\mathrm{CO}$. This low signal-to-noise gives rise to the unphysically large $V^{2}$ (i.e., normalized $V^{2}>1$ ) seen for $\lambda \gtrsim 2.33 \mu \mathrm{m}$.

The fluxes for V1515 Cyg appear reasonably consistent with our simple disk model (Figure 3 ). The $V^{2}$ and $\Delta \phi$ are not very consistent with the data, as seen in the high reduced $\chi^{2}$ value for the fit (Table 2). The large $\chi^{2}$ value is due, at least in part, to underestimated uncertainties. The large $V^{2}$ seen at the longwavelength edge of the band are unphysical, and so the plotted error bars are clearly underestimated. Over most of the band the model appears compatible with the noisy $V^{2}$ and $\Delta \phi$ data.

\section{CONCLUSIONS}

We presented near-infrared, spatially resolved, spectroscopic observations of FU Ori, V1057 Cyg, and V1515 Cyg, obtained with the KI. With $R=2000$ across the $K$ band, we spectrally resolved individual $\mathrm{CO}$ bandheads as well as the $\mathrm{Br} \gamma$ transition of hydrogen. Our measured fluxes, squared visibilities, and differential phases were compared with simple accretion disk models.

A disk model is consistent with the data for FU Ori, although we require some asymmetry in the inner $\sim 1$ AU of the disk to explain the measured, non-zero, differential phases. We speculate that the inner disk of FU Ori may be warped. The disk model also appears reasonably compatible with the data for V1515 Cyg, although these data are noisy and so the constraints are weak.

For V1057 Cyg the disk model does not fit our data well. The observed $\mathrm{CO}$ bandhead features are broader than expected for a disk in Keplerian rotation. The disk model also fails to reproduce compact $\mathrm{Br} \gamma$ emission seen in the data. We speculate that a rotating, high-velocity, inner disk wind may be contributing substantially to the observed near-IR emission in this source, at least at the epoch of our observations.

Overall this study suggests a more complex explanation for the near-IR observations of three canonical FU Ori stars than the unified picture of a simple accretion disk model. In addition to a rotating disk undergoing viscous accretion, outflow is also likely to be an important element in the interpretation of radiative and kinematic data. While the namesake object, FU Ori, is consistent with a simple accretion disk model, V1057 Cyg is not. It is possible that V1057 Cyg is undergoing an epoch of enhanced outflow and that other FU Ori objects experience similar episodes. However further observations, at additional epochs, are needed to test this hypothesis.

Data presented herein were obtained at the W. M. Keck Observatory, from telescope time allocated to the National Aeronautics and Space Administration through the agency's scientific partnership with the California Institute of Technology and the University of California. The Observatory was made possible by the generous financial support of the W. M. Keck Foundation. The authors wish to recognize and acknowledge the cultural role and reverence that the summit of Mauna Kea has always had within the indigenous Hawaiian community. We are most fortunate to have the opportunity to conduct observations from this mountain. The ASTRA program, which enabled the observations presented here, was made possible by funding from the NSF MRI grant AST-0619965. The Keck Interferometer is funded by the National Aeronautics and Space Administration as part of its Exoplanet Exploration program. This work has used software from NExSci at the California Institute of Technology. We are grateful to the ASTRA team for their support of the new instrumental mode used here and for their enthusiasm for the science enabled. We also thank R. Akeson, A. Ghez, R. MillanGabet, J. Monnier, and J.-U. Pott for useful comments on the manuscript. 


\section{REFERENCES}

Armitage, P. J., Livio, M., \& Pringle, J. E. 2001, MNRAS, 324, 705

Bell, K. R., \& Lin, D. N. C. 1994, ApJ, 427, 987

Biscaya, A. M., Rieke, G. H., Narayanan, G., Luhman, K. L., \& Young, E. T. 1997, ApJ, 491, 359

Calvet, N., Hartmann, L., \& Kenyon, S. J. 1993, ApJ, 402, 623

Carr, J. S., Harvey, P. M., \& Lester, D. F. 1987, ApJ, 321, L71

Clarke, C., Lodato, G., Melnikov, S. Y., \& Ibrahimov, M. A. 2005, MNRAS, 361, 942

Colavita, M. M., \& Wizinowich, P. L. 2003, Proc. SPIE, 4838, 79

Colavita, M., et al. 2003, ApJ, 592, L83

Croswell, K., Hartmann, L., \& Avrett, E. H. 1987, ApJ, 312, 227

Eisner, J. A., Lane, B. F., Hillenbrand, L., Akeson, R., \& Sargent, A. 2004, ApJ, 613,1049

Eisner, J. A., et al. 2010a, Proc. SPIE, 7734, 773411

Eisner, J. A., et al. 2010b, ApJ, 718, 774

Green, J. D., Hartmann, L., Calvet, N., Watson, D. M., Ibrahimov, M., Furlan, E., Sargent, B., \& Forrest, W. J. 2006, ApJ, 648, 1099

Greene, T. P., Aspin, C., \& Reipurth, B. 2008, AJ, 135, 1421

Hartmann, L., \& Calvet, N. 1995, AJ, 109, 1846

Hartmann, L., Hinkle, K., \& Calvet, N. 2004, ApJ, 609, 906

Hartmann, L., \& Kenyon, S. J. 1985, ApJ, 299, 462

Hartmann, L., \& Kenyon, S. J. 1987, ApJ, 312, 243

Hartmann, L., \& Kenyon, S. J. 1996, ARA\&A, 34, 207

Hauschildt, P. H., Allard, F., \& Baron, E. 1999, ApJ, 512, 377

Herbig, G. H. 1958, ApJ, 128, 259
Herbig, G. H. 1966, Vistas Astron., 8, 109

Herbig, G. H. 1977, ApJ, 217, 693

Herbig, G. H. 2009, AJ, 138, 448

Herbig, G. H., Petrov, P. P., \& Duemmler, R. 2003, ApJ, 595, 384

Kenyon, S. J., \& Hartmann, L. W. 1991, ApJ, 383, 664

Laugalys, V., \& Straižys, V. 2002, Balt. Astron., 11, 205

Lodato, G., \& Clarke, C. J. 2004, MNRAS, 353, 841

Malbet, F., et al. 2005, A\&A, 437, 627

Millan-Gabet, R., et al. 2006, ApJ, 641, 547

Miller, A. A., et al. 2011, ApJ, 730, 80

Pfalzner, S. 2008, A\&A, 492, 735

Pott, J., et al. 2010, ApJ, 721, 802

Quanz, S. P., Henning, T., Bouwman, J., Ratzka, T., \& Leinert, C. 2006, ApJ, 648,472

Racine, R. 1968, AJ, 73, 233

Reid, M. J., et al. 2009, ApJ, 700, 137

Reipurth, B., \& Aspin, C. 2004, ApJ, 608, L65

Reipurth, B., \& Aspin, C. 2010, in Evolution of Cosmic Objects through their Physical Activity, ed. H. A. Harutyunian, A. M. Mickaelian, \& Y. Terzian (Yerevan: Gitutyun), 19

Vorobyov, E. I., \& Basu, S. 2005, ApJ, 633, L137

Zhu, Z., Hartmann, L., Calvet, N., Hernandez, J., Muzerolle, J., \& Tannirkulam, A. 2007, ApJ, 669, 483

Zhu, Z., Hartmann, L., Calvet, N., Hernandez, J., Tannirkulam, A., \& D’Alessio, P. 2008, ApJ, 684, 1281

Zhu, Z., Hartmann, L., Gammie, C., \& McKinney, J. C. 2009, ApJ, 701, 620 